PSS PROCEEDINGS

\title{
Numerical study of the mass spectrum in the 2D $O(3)$ sigma model with a theta term
}

\section{B. Allés*}

INFN Sezione di Pisa, Pisa, Italy

E-mail: alles@df.unipi.it

\section{A. Papa}

Dipartimento di Fisica, Università della Calabria and INFN, Gruppo collegato di Cosenza Arcavacata di Rende, Cosenza, Italy

E-mail: papa@cs.infn.it

\begin{abstract}
It has been conjectured that the mass spectrum of the $\mathrm{O}(3)$ non-linear $\sigma$ model with a $\theta$ term in 2 dimensions may possess an excited state, which decays when $\theta$ is lowered from $\pi$ below a critical value. Since the direct numerical investigation of the model is prevented by a sign problem, we try to infer some information on the mass spectrum at real $\theta$ by studying the model at imaginary $\theta$ via analytic continuation. A modified Swendsen-Wang cluster algorithm has been introduced to simulate the model with the $\theta$ term.
\end{abstract}

The XXV International Symposium on Lattice Field Theory

July 30 - August 42007

Regensburg, Germany

\footnotetext{
${ }^{*}$ Speaker.
} 


\section{Introduction}

Integrable Quantum Field Theories in 2 dimensions are successfully studied by using the Smatrix approach both for massive [1] and massless theories [2,3]. On the other hand the study of non-integrable models is complicated because their scattering amplitudes are generally nonelastic. These models are in fact characterized by particle production, resonances, decay events, etc. along with the simple pole structure that features integrable theories.

The $\mathrm{O}(3)$ non-linear $\sigma$ model in 2 dimensions with a $\theta$ term is a well-known example of non-integrable theory. It is defined by the action [4]

$$
S=\frac{1}{2 g} \int \mathrm{d}^{2} x\left(\partial_{\mu} \vec{\phi}(x)\right)^{2}-i \theta \int \mathrm{d}^{2} x Q(x),
$$

where $g$ is the coupling constant, $\vec{\phi}(x)$ is a 3-component unit vector and $Q(x)$ is the topological charge density operator [5] ( $a, b, c$ are group indices that run from 1 to 3 and $\mu, v$ are 2-dimensional spacetime indices)

$$
Q(x)=\frac{1}{8 \pi} \varepsilon^{\mu v} \varepsilon_{a b c} \phi^{a}(x) \partial_{\mu} \phi^{b}(x) \partial_{v} \phi^{c}(x) .
$$

The integration of $Q(x)$ throughout the whole 2-dimensional spacetime yields the total topological charge $Q$ which takes on integer numbers that reveal the winding of configurations over the sphere $\mathrm{S}^{2}$. Topological charge (-)1 configurations are called (anti)instantons.

When $\theta=0$ or $\pi$ the model is integrable and its spectrum is as follows. For $\theta=0$ it contains a triplet of massive scalars whose mass has been analytically calculated [6] and numerically verified within a 2\%-3\% error [7]. At $\theta=\pi$ it has been conjectured that the theory becomes massless $[3,8]$ (for a numerical analysis of the corresponding universality class see [9]).

The study of the evolution of the spectrum as $\theta$ moves from $\pi$ towards lower values is worthwhile. The model contains a triplet and a singlet states whose masses ( $m_{T}$ and $m_{S}$ respectively) are proportional to $(\pi-\theta)^{2 / 3}$ for $0<(\pi-\theta) \ll 1[10]$. By using Form Factor Perturbation Theory [11] it has been shown that the singlet is heavier than the triplet [12] (see also Ref. [13]). In particular at $\theta \approx \pi$ the ratio of singlet to triplet masses is $m_{S} / m_{T} \approx \sqrt{3}$.

On the other hand it is known that at $\theta=0$ there is no lingering trace of the singlet state. This fact suggests that the singlet mass may diverge as $\theta$ approaches zero, thus decoupling from the whole theory. Consequently, by continuity arguments, it seems plausible that there exist a critical $\theta_{c}$ where the singlet mass becomes exactly twice the triplet mass in such a way that for all $\theta<\theta_{c}$ the singlet decays into states belonging to the triplet.

In Fig. 1 a sketch representing the qualitative behaviour expected for the $\theta$ dependence of the spectrum of the theory is shown. We are currently making a Monte Carlo study of the model to examine some of the salient features shown in this Figure. In the present progress report we give clear numerical indications that $m_{T}(\theta)$ vanishes at the endpoint $\theta_{\text {end }}=\pi$ (for an extensive account see [14]).

To this end we have prepared a code that simulates the 2-dimensional $\mathrm{O}(3)$ sigma model with an imaginary $\theta$ term. After extracting the triplet mass with this code, one can analytically continuate the results to real values of $\theta$ thus obtaining a numerical estimate of the lower curve in Fig. 1. We focus our attention on the location of the endpoint $\theta_{\text {end }}$ on this curve. In section 2 


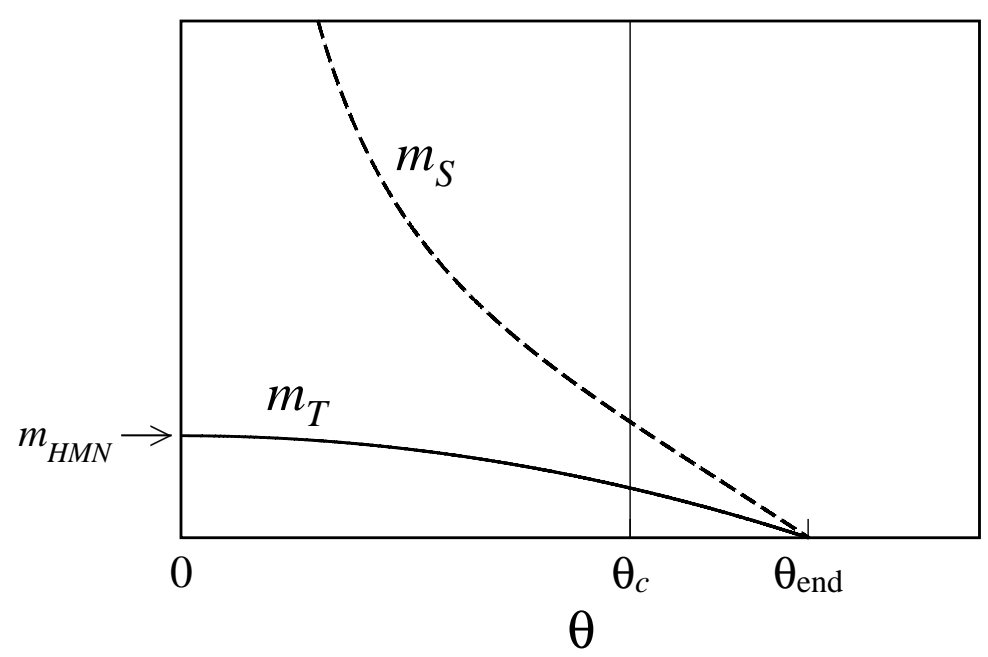

Figure 1: Sketch of the hypothesized $\theta$ dependence for the singlet (dashed line) and triplet (continuous line) masses in the $\mathrm{O}(3)$ non-linear sigma model in 2 dimensions. $\theta_{c}$ is the value where the singlet mass becomes larger than twice the triplet mass. $m_{H M N} \equiv m_{T}(\theta=0)$ is the mass calculated in Ref. [6]. $\theta_{\text {end }}$ is the endpoint where the theory becomes massless. It has been conjectured that $\theta_{\text {end }}=\pi$.

we shall shortly describe the updating algorithm used at imaginary $\theta$. In section 3 the analytic continuation is performed and the main results are exhibited.

\section{The Lattice implementation}

We have regularized the model in Eq.(1.1) by using the standard lattice action

$$
S_{L}=A_{L}-i \theta_{L} Q_{L}, \quad A_{L} \equiv-\beta \sum_{x, \mu} \vec{\phi}(x) \cdot \vec{\phi}(x+\widehat{\mu}), \quad Q_{L} \equiv \sum_{x} Q_{L}(x)
$$

where $\beta$ is the inverse bare lattice coupling constant for this standard regularization and $\theta_{L}$ is the bare theta parameter. The corresponding endpoint and critical point will be called $\theta_{L, \text { end }}$ and $\theta_{L, c}$. The lattice operator for the density of topological charge is defined as in [15],

$$
Q_{L}(x)=\frac{1}{32 \pi} \varepsilon^{\mu v} \varepsilon_{a b c} \phi^{a}(x)\left(\phi^{b}(x+\widehat{\mu})-\phi^{b}(x-\widehat{\mu})\right)\left(\phi^{c}(x+\widehat{v})-\phi^{c}(x-\widehat{v})\right) .
$$

The coordinate sites are labelled by $x \equiv\left(x_{1}, x_{2}\right)$. The total topological charge $Q_{L}$ obtained from the sum over the whole lattice volume of the above expression does not yield integer numbers on single configurations. This is not an inconvenience inasmuch as Quantum Field Theory deals with quantum averages of renormalized operators over many configurations. Thus the above definition for the density of topological charge (2.2) must be renormalized, the renormalized charge being $Q$

$$
Q_{L}=Z_{Q} Q
$$

and $Z_{Q}$ being the corresponding renormalization constant. It can be calculated either by perturbation theory [16] or by a non-perturbative numerical method [17]. The meaning of this constant 
is simple: it accounts for the average over quantum fluctuations in such a way that $Q$ yields an integer value. Actually this last observation is the basis for the above-mentioned non-perturbative numerical method (see below).

The reason to choose the expression (2.2) to be used in the lattice action is that, as we shall see later, it allows the introduction of a variant of the cluster algorithm in the presence of the $\theta$ term. There exist other lattice regularizations of the operator $Q(x)$ that do not require the computation of $Z_{Q}$ (since it is exactly 1 for all $\beta$ ); however it is not easy to introduce a fast cluster algorithm for them.

Our interest concerns the spectrum of the theory for varying $\theta$. This parameter is related to the corresponding bare $\theta_{L}$ by the expression $\theta=Z_{Q} \theta_{L}$. Therefore the value of the bare theta parameter $\theta_{L, \text { end }}$ where the mass vanishes is conjectured to be $\theta_{L, \text { end }}=\pi / Z_{Q}$. This expression is a function of $\beta$ since $Z_{Q}$ in general depends on the coupling constant.

We have run our Monte Carlo simulations at imaginary values of the theta parameter, $\theta_{L}=$ $+i \vartheta_{L}\left(\vartheta_{L} \in \mathbb{R}\right)$ in order to avoid the sign problem. Let us briefly introduce the new cluster algorithm for imaginary $\theta_{L}$.

The first part of an updating step with the Wolff algorithm [18] for the standard $\mathrm{O}(3)$ sigma model without a $\theta$ term consists in choosing a random unit vector $\vec{r}$ in such a way that every dynamical field can be split in a component parallel to $\vec{r}$ and the rest, $\vec{\phi}(x)=(\vec{\phi}(x) \cdot \vec{r}) \vec{r}+\vec{\phi}_{\perp}(x)$, where $\vec{\phi}_{\perp}(x)$ denotes the part of $\vec{\phi}(x)$ orthogonal to $\vec{r}$. Then the signs of $(\vec{\phi}(x) \cdot \vec{r})$ for all $x$ are updated à la Swendsen-Wang as in the Ising model.

By introducing the above separation for $\vec{\phi}(x)$ in the expression (2.2) we can rewrite it as

$$
\begin{aligned}
Q_{L}(x)=\frac{1}{16 \pi}\{ & (\vec{\phi}(x) \cdot \vec{r})\left(d_{1,2}+d_{-1,-2}+d_{2,-1}+d_{-2,1}\right)+ \\
& (\vec{\phi}(x+\widehat{1}) \cdot \vec{r})\left(d_{0,-2}-d_{0,2}\right)+(\vec{\phi}(x-\widehat{1}) \cdot \vec{r})\left(d_{0,2}-d_{0,-2}\right)+ \\
& \left.(\vec{\phi}(x+\widehat{2}) \cdot \vec{r})\left(d_{0,1}-d_{0,-1}\right)+(\vec{\phi}(x-\widehat{2}) \cdot \vec{r})\left(d_{0,-1}-d_{0,1}\right)\right\},
\end{aligned}
$$

where $x \pm \hat{1}$ means the site at the position one step forward (backward) in the direction " 1 " starting from site $x$ and the notation $d_{i, j}$ stands for the $3 \times 3$ determinant (the three components for each vector are unfold along the rows)

$$
d_{i, j} \equiv \operatorname{det}\left(\begin{array}{c}
\vec{r} \\
\vec{\phi}(x+\widehat{i}) \\
\vec{\phi}(x+\widehat{j})
\end{array}\right) .
$$

In this fashion the theory at each updating step looks like an Ising model in the presence of an external local magnetic field $h(x)$ because the $\theta$ term in Eq.(2.4) is linear in $(\vec{\phi} \cdot \vec{r})$. One can readily derive that the magnetic field at site $x$ is

$$
\begin{aligned}
h(x)=-\frac{\vartheta_{L}}{16 \pi}|\vec{\phi}(x) \cdot \vec{r}| & \left(d_{1,2}+d_{-1,-2}+d_{2,-1}+d_{-2,1}+d_{-1,-1-2}+d_{-1+2,-1}+\right. \\
& \left.d_{1,1+2}+d_{1-2,1}+d_{2,2-1}+d_{2+1,2}+d_{-2,-2+1}+d_{-2-1,-2}\right) .
\end{aligned}
$$

$d_{i+k, j}$ (and analogous terms in (2.6)) are the straightforward generalization of the above definition (2.5) when the site is obtained by shifting two steps ( $\widehat{i}$ plus $\widehat{k}$ ) from the original position $x$. 


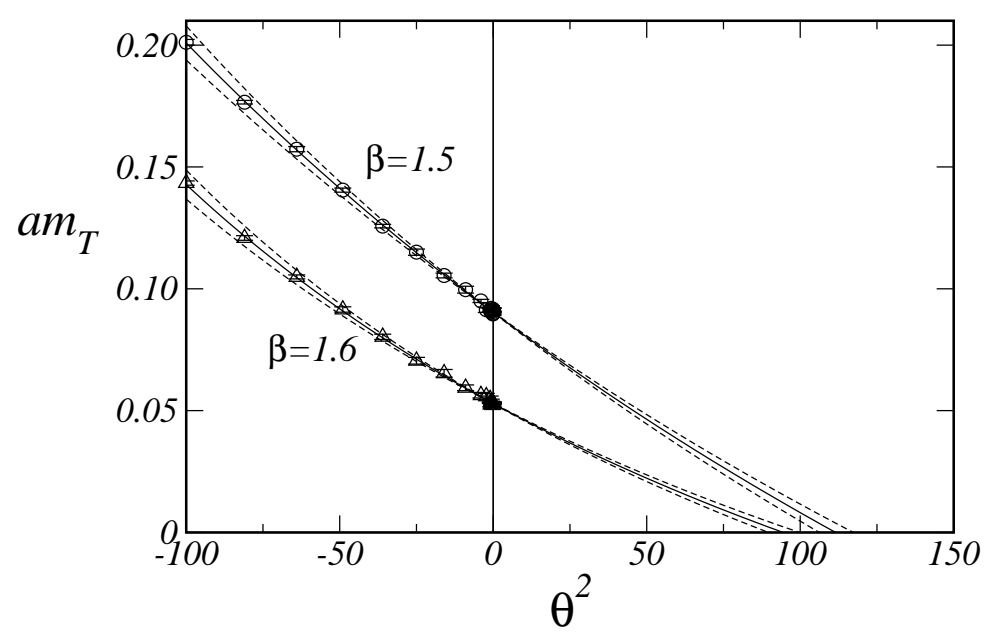

Figure 2: Behaviour of the triplet mass (in units of the lattice spacing) as a function of $\theta^{2}$. Circles $(\beta=1.5)$ and triangles $(\beta=1.6)$ are the data from the simulation at imaginary $\theta\left(\theta^{2}<0\right)$. Each continuous line is the result of the analytic continuation described in the text and the dashed lines enclose the boundary of its error.

We have converted the original theory in an Ising model in the presence of a local external magnetic field (which changes at each updating step and therefore must be recalculated at each step). In the literature there are two algorithms expressly introduced to update Ising models in the bosom of magnetic fields: the Lauwers-Rittenberg [19] and the Wang [20] methods. We tested the performances of both algorithms, compared their decorrelation times and decided for the latter. The cluster construction was tackled by the Hoshen-Kopelman algorithm [21].

\section{Analytic continuation and determination of $\theta_{\text {end }}$}

Operators which couple the vacuum with the singlet or triplet states can contain an arbitrary number of fundamental fields since the model is not parity invariant for $\theta \neq 0$. We only present the results for the triplet particle. The following operators were used

$$
\overrightarrow{\mathscr{O}}_{1}(x) \equiv \vec{\phi}(x), \quad \overrightarrow{\mathscr{O}}_{2}(x) \equiv-i \vec{\phi}(x) \times \vec{\phi}(x+\widehat{1}) .
$$

Then we calculated the related wall operators by averaging over the $x_{1}$ coordinate (as usual $L$ is the lattice size), $\overrightarrow{\mathscr{W}}_{i}\left(x_{2}\right) \equiv \frac{1}{L} \sum_{x_{1}} \overrightarrow{\mathscr{O}}_{i}(x)$ for $i=1,2$.

To single out the correct parity mixture for the physical particle and to clean the signal from possible excited states, we extracted the triplet mass $m_{T}$ by using the variational method of Ref. [22] where the mass is obtained from the largest eigenvalue of the correlation matrix $\left\langle\overrightarrow{\mathscr{W}}_{i}\left(x_{2}\right) \overrightarrow{\mathscr{W}}_{j}(0)\right\rangle-$ $\left\langle\overrightarrow{\mathscr{W}}_{i}\right\rangle\left\langle\overrightarrow{\mathscr{W}}_{j}\right\rangle$. In Fig. 2 the results for the triplet mass are shown for two values of $\beta$. The analytic continuations in this figure were done by using a ratio of degree 2 polynomials; analogous results are obtained from other functional forms (we checked this statement by using degree 4 and 6 polynomials). The endpoint (where the mass vanishes) equals $\left(\theta_{L \text {,end }}\right)^{2}=\left(\theta_{\text {end }} / Z_{Q}(\beta)\right)^{2}$ where $\theta_{\text {end }}$ is conjectured to be equal to $\pi$. On the other hand the renormalization constant $Z_{Q}$ can be evaluated 


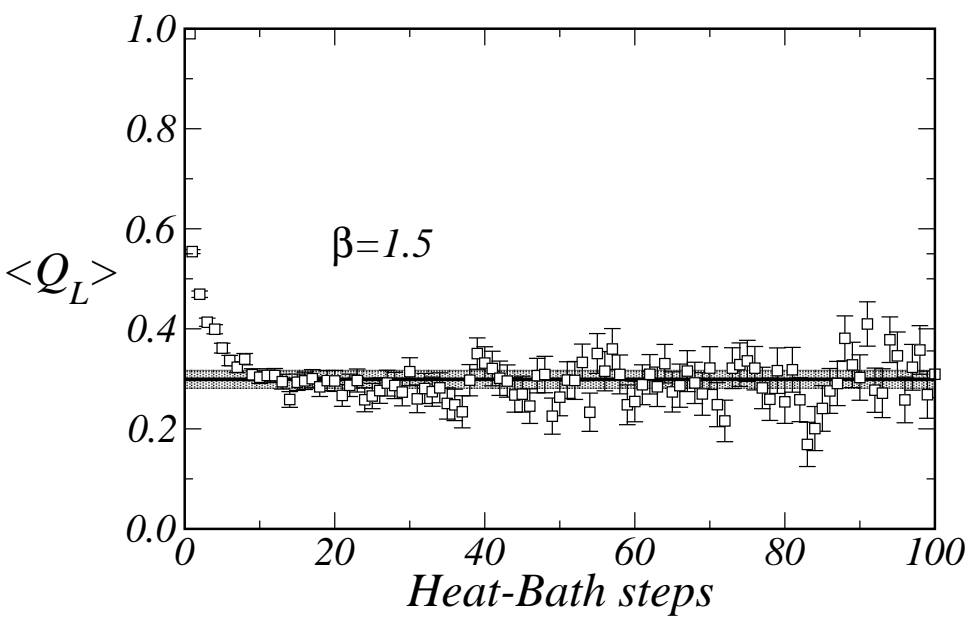

Figure 3: Data for $\left\langle Q_{L}\right\rangle$ start at +1 at the 0-th Heat-Bath step and then they go down until reaching a plateau. The horizontal line and grey band are the value and error respectively of $Z_{Q}(\beta=1.5)$.

with the non-perturbative numerical method of Ref. [17]. An example of such an evaluation is shown in Fig. 3. Summed up briefly: a classical instanton (with topological charge +1 ) is put by hand on the lattice and then it is heated by applying 100 updating steps (we used Heat-Bath steps on the conventional $\mathrm{O}(3)$ non-linear sigma model without a $\theta$ term since $Z_{Q}$ cannot depend on $\theta$ ). The value of $Q_{L}$ is measured while the continuum topological charge $Q$ is continuously monitored (by 6 cooling steps after every Heat-Bath updating) to be sure that the background charge is not changed. This procedure was repeated $10^{4}$ times for both values of $\beta$. The average of $Q_{L}$ on configurations that lie in the topological sector +1 yields $Z_{Q}$.

Table 1

\begin{tabular}{cclll}
\hline \hline$\beta$ & $L$ & $\left(\theta_{L, \text { end }}\right)^{2}$ & $Z_{Q}$ & $\theta_{\text {end }}$ \\
\hline 1.5 & 120 & $111(5)$ & $0.299(18)$ & $3.15(20)$ \\
\hline 1.6 & 180 & $94(5)$ & $0.313(12)$ & $3.03(14)$ \\
\hline \hline
\end{tabular}

Then, once we know $Z_{Q}$ and $\left(\theta_{L, \text { end }}\right)^{2}$ the value of $\theta_{\text {end }}$ can be extracted. The results are shown in Table 1. The lattice sizes were chosen large enough to guarantee the absence of relevant finite size effects (we imposed $L / \xi \equiv L \cdot a m_{T} \geq 10$ ). $2 \cdot 10^{5}$ propagators were measured on as many independent configurations for all values of $\beta$ and $\theta_{L}$. The results of the last column are in fair agreement with the conjecture that predicts $\theta_{\text {end }}=\pi$. By averaging over the results for both $\beta$ (assuming gaussian errors) we obtain that the endpoint for the triplet mass is $\theta_{\text {end }}=3.07(11)$.

\section{Conclusions and outlook}

We are studying the spectrum of the $2 \mathrm{D} \mathrm{O}(3)$ non-linear sigma model with a $\theta$ term as a function of this parameter. The model contains a triplet and a singlet states whose mass depend on $\theta$ as shown in the sketch of Fig. 1. In the present progress report we have given clear evidence 
that the triplet mass indeed vanishes when $\theta$ becomes $\pi$. To do so, we have simulated the model at imaginary $\theta$ and then extrapolated the results to real $\theta$. The extrapolation always indicate that the mass tends to vanish at an endpoint. Our calculation yields $\theta_{\text {end }}=3.07(11)$ in good agreement with the conjectured prediction $\theta_{\text {end }}=\pi$.

A new fast cluster algorithm was purposely introduced to simulate the theory at imaginary $\theta$.

We are planning to improve the statistics by studying the model at other values of $\beta$ and other lattice regularizations of the topological charge as well as by increasing the precision in the evaluation of $Z_{Q}$ which is the largest source of error in the calculation of $\theta_{\text {end }}$ (see Ref. [14]). We will also extend the analysis to the singlet mass and the determination of $\theta_{c}$.

We emphasize the good performance of the analytic continuation in our study. It is important for that to have got Monte Carlo data within a wide range of values of $\theta_{L},\left(\vartheta_{L} \equiv-i \theta_{L} \in[0,10]\right)$.

\section{References}

[1] A. B. Zamolodchikov, Al. B. Zamolodchikov, Ann. Phys. NY 120, 253 (1979).

[2] Al. B. Zamolodchikov, Nucl. Phys. B358, 524 (1991).

[3] A. B. Zamolodchikov, Al. B. Zamolodchikov, Nucl. Phys. B379, 602 (1992).

[4] A. M. Polyakov, Phys. Lett. B59, 79 (1975); E. Brézin, J. Zinn-Justin, Phys. Rev. B14, 3110 (1976).

[5] A. A. Belavin, A. M. Polyakov, JETP Letters 22, 245 (1975).

[6] P. Hasenfratz, M. Maggiore, F. Niedermayer, Phys. Lett. B245, 522 (1990).

[7] B. Allés, A. Buonanno, G. Cella, Nucl. Phys. B500, 513 (1997).

[8] I. Affleck, F. D. M. Haldane, Phys. Rev. B36, 5291 (1977); R. Shankar, N. Read, Nucl. Phys. B336, 457 (1990).

[9] W. Bietenholz, A. Pochinsky, U.-J. Wiese, Phys. Rev. Lett. 75, 4524 (1995); V. Azcoiti, G. Di Carlo, A. Galante, Phys. Rev. Lett. 98, 257203 (2007).

[10] I. Affleck, D. Gepner, H. J. Schulz, T. Ziman, J. Phys. A 22, 511 (1989).

[11] G. Delfino, G. Mussardo, P. Simonetti, Nucl. Phys. B473, 469 (1996).

[12] D. Controzzi, G. Mussardo, Phys. Rev. Lett. 92, 021601 (2004); Phys. Lett. B617, 133 (2005).

[13] L. Campos Venuti et al., J. Stat. Mech. 0504, L02004 (2005).

[14] B. Allés, A. Papa, arXiv:0711.1496 [cond-mat].

[15] A. Di Giacomo, F. Farchioni, A. Papa, E. Vicari, Phys. Rev. D46, 4630 (1992).

[16] M. Campostrini, A. Di Giacomo, H. Panagopoulos, Phys. Lett. B212, 206 (1988).

[17] A. Di Giacomo, E. Vicari, Phys. Lett. B275, 429 (1992); B. Allés, M. Campostrini, A. Di Giacomo, Y. Gündüç, E. Vicari, Phys. Rev. D48, 2284 (1993); F. Farchioni, A. Papa, Nucl. Phys. B431, 686 (1994).

[18] R. Swendsen, J.-S. Wang, Phys. Rev. Lett. 58, 86 (1987); U. Wolff, Phys. Rev. Lett. 62 , 361 (1989).

[19] P. G. Lauwers, V. Rittenberg, Phys. Lett. B233, 197 (1989).

[20] J.-S. Wang, Physica A161, 249 (1989).

[21] J. Hoshen, R. Kopelman, Phys. Rev. B14, 3438 (1976).

[22] A. S. Kronfeld, Nucl. Phys. (Proc. Suppl.) 17, 313 (1990); M. Lüscher, U. Wolff, Nucl. Phys. B339, 222 (1990). 